\title{
QUANTITATIVE POLYNOMIAL APPROXIMATION ON CERTAIN PLANAR SETS $\left({ }^{1}\right)$
}

\author{
BY \\ D. J. NEWMAN AND L. RAYMON
}

I. Let $Q$ be a compact space in $E^{k}$. The massivity, $m_{n}(Q)$, is a sequence defined as follows: Let $X_{n}$ be a set of $n+1$ elements of $Q$; then

$$
m_{n}(Q)=\max _{X_{n} \subset Q} \min _{x_{i}, x_{j} \in X_{n} ; i \neq j}\left|x_{i}-x_{j}\right| .
$$

Note that $m_{1}$ is what is normally defined to be the diameter of the space. Also note that $m_{n}$ decreases to zero monotonely as $n \rightarrow \infty$. E.g., if $Q$ is a set of measure $m>0, m_{n}(Q)$ is asymptotic to $m / n$; if $Q$ is a $k$-dimensional cube, $m_{n}(Q)$ is asymptotic to $c / n^{1 / k}[6$, p. 21$]$.

$Q_{1}$ will represent the interval $0 \leqq x \leqq 1$, and $Q_{2}$ the square $0 \leqq x, y \leqq 1$. Given a function $f(x)$ defined on $Q$ and a $\delta>0$, the modulus of continuity of the function $f(x), \omega_{f}(\delta)$, is defined as follows:

$$
\omega_{f}(\delta)=\sup _{|x-y| \leqq \delta}|f(x)-f(y)| .
$$

For $f(x)$ in $L^{2}\left(Q_{1}\right)$, we continue $f(x)$ to have period 1 , and we define

$$
\omega_{f}(\delta)=\sup _{|t| \leqq \delta}\left(\int_{0}^{1}|f(x+t)-f(x)|^{2} d x\right)^{1 / 2},
$$

while for $f(x, y)$ in $L^{2}\left(Q_{2}\right)$ continued to have period 1 in $x$ and in $y$

$$
\omega_{f}(\delta)=\sup _{t^{2}+s^{2} \leqq \delta^{2}}\left(\int_{0}^{1} \int_{0}^{1}|f(x+t, y+s)-f(x, y)|^{2} d x d y\right)^{1 / 2} .
$$

$\omega(\delta)$ is a nonnegative, nondecreasing continuous function of $\delta . f(x)$ is continuous iff $\omega_{f}\left(0^{+}\right)=0$. Also, $\omega(\delta)$ is sublinear, and hence $\omega_{f}(\delta) \geqq c \delta$ for some constant $c$. If $\omega_{f}(\delta) \leqq \delta$ for all $\delta>0, f(x)$ is said to be a contraction on $Q$.

Let $C(Q)$ be the space of all real valued continuous functions on $Q$ and let $P$ be a finite dimensional subspace. Let $K$ be the class of contractions on $Q$. We now introduce the degree of approximation

$$
\rho_{P}=\sup _{f \in K} \inf _{p \in P}\|f-p\|
$$

Presented to the Society, January 24, 1968; received by the editors March 7, 1967 and, in revised form, August 2, 1967.

( ${ }^{1}$ This research was partially supported by the National Science Foundation, Grant GP-4391. 
We have the following general lower bound theorem for the degree of approximation: If the dimension of $P$ is $n$, then $\rho_{P} \geqq m_{n}(Q) / 2$ [4]. If for some $Q$ there exists $c$ such that $\rho \leqq c m_{n}(Q)$ for all $n$, where $P$ is the class of $n$th degree polynomials, we can then say that $P$ efficiently approximates all contractions on $Q$. If for some family of compact sets $Q_{\zeta}$ there exists $c$ such that $\rho_{P}(Q) \leqq c m_{n}(Q)$ for all $Q$ in $Q_{\zeta}$ and for all $n$, then $P$ can be said to approximate contractions efficiently on the family $Q_{\zeta}$.

The classic upper bound theorem is that of Dunham Jackson which says, essentially, that polynomials approximate efficiently on $Q_{1}[3$, p. 36]. More recently, further results in this direction have been obtained. Yu. A. Brudnji [2] and D. J. Newman simultaneously discovered that for $P_{n}$ the class of $n$th degree polynomials on linear sets $Q$ of positive measure, the corresponding degree of approximation, $\rho_{n}$, for $n$ sufficiently large, satisfies the inequality $\rho_{n} \leqq c m / n, c$ independent of $Q$. Newman and H. S. Shapiro have shown that polynomials approximate efficiently on the family of cubes, spheres and balls of all dimensions [6]. On the other hand, polynomials are not efficient on the family of all rectangles in the plane (and, hence, the two-dimensional analog of the result for linear sets of positive measure cannot hold). In fact, there is even a (highly pathological) linear set (of measure zero) on which polynomials are not efficient.

An $n$th degree polynomial in more than one variable will refer to a polynomial in which the maximum degree in any single variable is less than $n$. Unless otherwise specified, $P$ and the corresponding $\rho$ will refer to $n$th degree polynomials. In this case, they will sometimes be denoted, respectively, $P_{n}$ and $\rho_{n}$. (Note that $P_{n}$ may have dimension greater than $n$.)

The term normal curve will be used for a compact continuous curve of finite length contained in $Q_{2}$. In this article we seek estimates for $\rho_{P}(Q)$ for $Q$ a normal curve.

II. Lemma 1. If $Q$ is a normal curve, there exist $c_{1}, c_{2}>0$ such that $c_{1} / n \leqq m_{n}(Q)$ $\leqq c_{2} / n$.

Proof. The projection of $Q$ on the $x$-axis (or the $y$-axis) must be a line segment $[a, b]$. Consider the points $\{a+k(b-a) / n, k=0,1, \ldots, n\}$. Invert back to $n+1$ corresponding points of $Q$ to get the set $X_{n}$. The $x$-coordinate (or the $y$-coordinate) of the minimum distance between any two points of $X_{n}$ is $(b-a) / n$, giving $m_{n}(Q) \geqq(b-a) / n$. Also, $m_{n}(Q) \leqq 2 L / n$, where $L$ is the length of $Q$. For, otherwise, there would be $n+1$ nonintersecting discs of radius $L / n$, with their centers being elements of $Q$. The portion of $Q$ contained within these discs would have length $\geqq 2 L$. This contradiction establishes Lemma 1 .

LEMMA 2. On $Q_{2}$, there exist $c_{1}, c_{2}>0$ such that $c_{1} / n<\rho_{n}<c_{2} / n$.

This lemma is a special case of the more general theorem of Newman and Shapiro [6, Theorem 4, p. 212]. 
LEMMA 3. Let $f(x)$ be a function defined on $S \subset Q, Q$ a compact set in $E^{n}$, where $f(x)$ has modulus of continuity $\omega(\delta)$. Then there is a function $f^{*}(x)$ defined on $Q$ with the following properties:

(a) $f^{*}(x)=f(x), x \in S$,

(b) $\left|f^{*}(x)-f^{*}(y)\right| \leqq \omega(d(x, y))$, where $d(x, y)$ is the distance from $x$ to $y$. (In other words, a function can be extended without changing its modulus of continuity.)

Proof. It is sufficient to show that $f$ can be properly extended to one point $\bar{x}$ not in $S$, for the result would then follow by transfinite induction.

For all $x, y$ in $S$,

$$
\begin{aligned}
f(x)-f(y) & \leqq \omega(d(x, y)) \\
& \leqq \omega(d(x, \bar{x})+d(y, \bar{x})), \text { by the triangle inequality and by the } \\
& \text { fact that } \omega \text { is nondecreasing, } \\
& \leqq \omega(d(x, \bar{x}))+\omega(d(y, \bar{x})), \text { by sublinearity of } \omega .
\end{aligned}
$$

Hence, for any $x$ we can find $\alpha$ so that

$$
f(x)-\omega(d(x, \bar{x})) \leqq \alpha \leqq f(x)+\omega(d(x, \bar{x})) .
$$

Defining $f^{*}(\bar{x})$ to be $\alpha$, we have

$$
|f(x)-f(\bar{x})| \leqq \omega(d(x, \bar{x})) .
$$

COROLlaRY. Let $f$ be a contraction on $S \subset Q, Q \subset E^{n}$. Then $f$ can be extended as a contraction on $Q$.

LeMmA 4. The binomials $x^{i} y^{j}, 0 \leqq i, j \leqq n$, generate on the curve $\sum_{k=0 ; m=0}^{N} a_{k m} x^{k} y^{m}$ $=0$ a vector space of dimension less than $3 \mathrm{Nn}$.

Proof. Let $S$ be the space generated by the $x^{i} y^{j}, 0 \leqq i, j \leqq n$ on the entire plane. Consider the space generated by the $x^{i} y^{j}, 0 \leqq i, j \leqq n$ on the restriction to the given algebraic curve. A basis of the binomials for this space spans, on the plane, a subspace $S_{0}$ of $S$. Another subspace $S_{1}$ of $S$ is generated by those binomials spanning $S$ which are not included in the basis for $S_{0}$. Note that the dimension of $S$ is $(n+1)^{2}$. We must show that $\operatorname{dim} S_{0}<3 N n$. For $n<N$, the result is trivial. Now,

$$
x^{i} y^{j} \sum_{0}^{N} a_{k m} x^{k} y^{m}, \quad 0 \leqq i, j \leqq n-N,
$$

are linearly independent elements of $S_{1}$. Hence $\operatorname{dim} S_{1} \geqq(n-N)^{2}$. Thus

$$
\operatorname{dim} S_{0}=\operatorname{dim} S-\operatorname{dim} S_{1} \leqq(n+1)^{2}-(n-N)^{2}<3 n N, \quad n \geqq N,
$$

and the lemma is proved.

LEMMA 5. If $Q$ is a normal curve, then there exist $c_{1}, c_{2}>0$ such that $c_{1} / n^{2}<\rho_{n}$ $<c_{2} / n$ for all $n$. 
Proof. Since the dimension of the space spanned by $n$th degree polynomials on $Q$ is $\leqq n^{2}$, we have $\rho_{n} \geqq m_{n^{2}} / 2 \geqq c_{1} / n^{2}$, by the general lower bound theorem and Lemma 1 . To prove the right hand inequality, we can consider any contraction on $Q$ to be extended, by the corollary to Lemma 3, as a contraction to $Q_{2}$. Now $\rho_{n}(Q) \leqq \rho_{n}\left(Q_{2}\right) \leqq c_{2} / n^{2}$, by Lemma 2 , completing the proof.

THEOREM 1. If $Q$ is the restriction of an algebraic curve

$$
\sum_{i=0 ; j=0}^{N} a_{i j} x^{i} y^{j}=0
$$

to $Q_{2}$, then there exist $c_{1}, c_{2}>0$ such that $c_{1} / n \leqq \rho_{n}(Q) \leqq c_{2} / n$.

Proof. From Lemma 4, the general lower bound theorem and Lemma 1, $\rho_{n} \geqq 1 / 6 N n$, while $\rho_{n} \leqq c / n$ by Lemma 5 .

For $Q$ a normal curve, the trivial estimates of $c_{1} / n$ and $c_{2} / n^{2}$ as upper and lower bounds for $\rho_{n}$ have been established. If the dimension of $n$th degree polynomials on $Q$ is equal to $O(n)$, then $c_{1} / n<\rho_{n}<c_{2} / n$ (as in the case where $Q$ is an algebraic curve). If the dimension of $n$th degree polynomials on $Q$ is of greater order than $n$, say of order $n^{2}$, to what extent can the trivial estimates for $\rho_{n}$ be improved? Theorem 2 and Theorem 5 provide a partial answer to this question. It is toward the establishment of these theorems that we conclude this section with several additional lemmata. In Theorem $1 \rho_{n}$ can be taken in the $L^{2}$ norm or the sup norm, where what is meant by $\|f\|_{L^{2}}$ on the normal curve $y=g(x)$ is $\left(\int_{0}^{1}|f(x, g(x))|^{2} d x\right)^{1 / 2}$. In all following results $\rho_{P}$ will be considered in $L^{2}$ only.

We denote by $C_{P}$ the set of all $\varphi(x)$ with $L^{2}[0,1]$ norm one which are in the orthogonal complement of $P$.

LEMMA 6.

$$
\rho_{P}=\sup _{\varphi \in C_{P}}\left\|\int_{0}^{x} \varphi(t) d t\right\|_{L^{2}} \cdot
$$

For proof, cf. [5, Lemma 2, p. 942].

We recall that $F(x)$ is said to be in the Paley-Wiener class for the upper half plane, $P W$, if

(a) $F$ is analytic in the upper half plane, and

$$
\int_{-\infty}^{\infty}|F(x+i y)|^{2} d x<M \quad \text { for all } y>0
$$

or, equivalently,

$$
F(z)=\int_{0}^{\infty} e^{i z x} \varphi(x) d x, \quad \varphi(x) \in L^{2}[0, \infty]
$$

For proof of the equivalence of (a) and (b), and for the general Paley-Wiener theory, the reader is referred to [7, pp. 1-13]. 
Lemma 7. Let $P=\left\{x^{\lambda}, \lambda \in \Lambda\right\}$, where $0 \in \Lambda, \lambda \geqq 0$. Then

$$
\rho_{P}^{2}\left(Q_{1}\right)=\sup _{F \in P W} \frac{\int_{-\infty}^{\infty} \frac{|F(x+i)|^{2}}{x^{2}+1 / 4} \prod_{\lambda \in \Lambda} \frac{x^{2}+(\lambda-1 / 2)^{2}}{x^{2}+(\lambda+3 / 2)^{2}} d x}{\int_{-\infty}^{\infty}|F(x)|^{2} d x} .
$$

For proof, cf. [5, Lemma 3, p. 943].

It is convenient to adopt the following notation:

$$
M(\Lambda)=\max _{x} \frac{1}{x^{2}+1 / 4} \prod_{\lambda \in \Lambda} \frac{x^{2}+(\lambda-1 / 2)^{2}}{x^{2}+(\lambda+3 / 2)^{2}}
$$

LeMma 8. For $P$ defined as in Lemma $7, \rho_{P}^{2}\left(Q_{1}\right) \leqq M(\Lambda)$.

Since, by Parseval's Identity,

$$
\int_{-\infty}^{\infty}|F(x+i)|^{2} d x \leqq \int_{-\infty}^{\infty}|F(x)|^{2} d x, \quad \text { for all } F \in P W,
$$

Lemma 8 is a corollary to Lemma 7.

LEMmA 9. If $\left|\sum_{k=0}^{n} a_{k} x^{k}\right| \leqq 1$ whenever $0 \leqq x \leqq 1$, then

$$
\left|a_{k}\right| \leqq 2^{2 k} \frac{n(n+k-1) !}{(n-k) !(2 k) !}
$$

Furthermore, these are the best possible bounds for $\left|a_{k}\right|$.

To derive this bound, one demonstrates that $\cos 2 n\left(\arccos x^{1 / 2}\right)$, which is equal to

$$
\sum(-1)^{k} 2^{2 k} \frac{n(n+k-1) !}{(n-k) !(2 k) !} x^{k}
$$

is maximal for each coefficient. For proof, see [1, p. 30]. Note that the above upper bounds yield the estimate $\left|a_{k}\right| \leqq 3^{2 n}$ for all $k$.

LEMMA 10. If $\left|\sum_{k=0}^{n^{2}} a_{k} x^{k / n}\right| \leqq 1,0<\delta \leqq x \leqq 1$, then

$$
\left|a_{k}\right| \leqq\left(\frac{3}{1-\delta}\right)^{2 n^{2}}, \quad k=0,1, \ldots, n^{2} .
$$

Proof. Assume $\left|\sum_{0}^{n^{2}} a_{k} w^{k}\right| \leqq 1,0<\delta \leqq w \leqq 1$. Let $y=(w-\delta) /(1-\delta)$. Then

$$
\left|\sum_{0}^{n^{2}} a_{k}((1-\delta) y+\delta)^{k}\right|=\left|\sum b_{k} y^{k}\right| \leqq 1, \quad 0 \leqq y \leqq 1 .
$$

Then, by Lemma $9,\left|b_{k}\right| \leqq 3^{2 n^{2}}$. This, in turn, gives

$$
\left|a_{k}\right|<\left(\frac{3}{1-\delta}\right)^{2 n^{2}} \text {. }
$$

Letting $w=x^{1 / n}$, the result follows. 
LEMMA 11. Let $p, q, n$ be integers, $p<q \leqq n$, with $p$, q relatively prime. The inequality $k q<p m_{1}+q m_{2}<(k+1) q, m_{1}, m_{2}, k$ positive integers, has at least $k$ solutions in $m_{1}, m_{2}$ whenever $k \leqq q-1$. For $q-1<k \leqq n$, the inequality has $q-1$ solutions.

Proof. The solutions in $m_{1}, m_{2}$ of $k q<p m_{1}+q m_{2}<(k+1) q$ are the same as the solutions in $m_{1}, m_{2}$ of $k<m_{1} p / q+m_{2}<k+1 . m_{1} p / q$ is a nonintegral rational which is less than $k$ for any $m_{1}$ in $\{1,2, \ldots, k\}$. Thus, letting $m_{2}=1+\left[k-m_{1} p / q\right]$, there is exactly one solution for $m_{1}=1,2, \ldots, k$, hence at least $k$ solutions.

Similarly, for $k \geqq q, m_{1} p / q+1+\left[k-m_{1} p / q\right]$ are solutions for $m=1,2, \ldots, q-1$, and the lemma is proved.

III. Our main theorems are Theorem 2 and Theorem 5.

THEOREM 2. If $Q$ is the set $\left(x, e^{x}\right), 0 \leqq x \leqq 1$, then there exist $c_{1}, c_{2}>0$ such that

$$
\frac{c_{1}}{n^{3 / 2}} \leqq \max _{f \in k} \min _{p \in P_{n}}\left\|f\left(x, e^{x}\right)-p\left(x, e^{x}\right)\right\|_{L^{2}} \leqq \frac{c_{2}}{n^{3 / 2}},
$$

i.e., $c_{1} / n^{3 / 2} \leqq \rho_{n} \leqq c_{2} / n^{3 / 2}$ in the $L^{2}$ norm.

Let $\alpha$ be an irrational number. $\alpha$ will be called of degree $f(n)$ if there exist $p, n$ such that $|\alpha-p / n|<1 / f(n)$ for infinitely many $n$.

THEOREM 5. Let $\alpha, 0<\varepsilon \leqq \alpha \leqq 1$ be an irrational of degree $n^{4}(3 /(1-\delta))^{2 n^{2}}$, $0<\delta<1$. Let $Q$ be the set of points $\left(x, x^{\alpha}\right), \delta \leqq x \leqq 1$. Then there exists a subsequence $\rho_{n_{1}}$ of $\rho_{n}$ and constants $c_{1}, c_{2}>0$ such that, in the $L^{2}$ norm, $c_{1} / n_{i}^{3 / 2} \leqq \rho_{n_{i}} \leqq c_{2} / n_{i}^{3 / 2}$.

Proof of Theorem 2. We are approximating with linear combinations of $x^{k} e^{m x}, k, m \leqq n$. By Lemma 6 ,

$$
\rho_{n}=\sup _{\varphi \in C_{P}}\left\|\int_{0}^{x} \varphi(t) d t\right\|, \quad \text { where } \int_{0}^{1} \varphi(x) x^{k} e^{m x} d x=0, \quad k, m \leqq n,
$$

or, letting $t e=e^{x}$, where

$$
\int_{1 / e}^{1} \varphi(t)(\log t)^{k} t^{m-1} d t=0, \quad k, m \leqq n .
$$

Let

$$
F(z)=\int_{1 / e}^{1} \varphi(t) t^{-(i z+1 / 2)} d t
$$

Letting $t=e^{-u}$, we note that $F \in P W$. Setting $k=0$ in (1), we get $F\left(\left(m+\frac{1}{2}\right) i\right)=0$, $m \leqq n-1$. Setting $k=1$ in (1) and integrating by parts, we get $F^{\prime}\left(\left(m+\frac{1}{2}\right) i\right)=0$, $m \leqq n-1$. For general $k$, integration by parts $k$ times yields

$$
F^{(k)}\left(\left(m+\frac{1}{2}\right) i\right)=0, \quad m \leqq n-1 .
$$

(In each case the boundary terms drop out by the orthogonality of $\varphi$ to $x^{k} e^{m x}$.) 
Since $F(x)=\int_{-\infty}^{\infty} e^{i u x} e^{-u / 2} \varphi\left(e^{-u}\right) d u$, Parseval's Identity yields

$$
\frac{1}{2 \pi} \int_{-\infty}^{\infty}|F(x)|^{2} d x=\int_{-\infty}^{\infty} e^{-u}\left|\varphi\left(e^{-u}\right)\right|^{2} d u=\int_{0}^{1}|\varphi(t)|^{2} d t=1
$$

Integrating by parts,

$$
F(z)=t^{-(i z+1 / 2)} \int_{0}^{t} \varphi(u) d u+(i z+1 / 2) \int_{1 / e}^{1} t^{-(i z+3 / 2)} \int_{0}^{t} \varphi(u) d u d t .
$$

But $\varphi$ is orthogonal to 1 , hence

Thus

$$
F(z)=(i z+1 / 2) \int_{1 / e}^{1} t^{-(i z+3 / 2)} \int_{0}^{t} \varphi(u) d u d t
$$

$$
\begin{aligned}
\frac{F(x+i)}{i x+1 / 2} & =\int_{1 / e}^{1} t^{-(i x+1 / 2)} \int_{0}^{t} \varphi(u) d u d t \\
& =\int_{-\infty}^{\infty} e^{i v x-v / 2} \int_{0}^{e^{-v}} \varphi(u) d u d v .
\end{aligned}
$$

Hence, by Parseval's Identity,

(4) $\frac{1}{2 \pi} \int_{-\infty}^{\infty} \frac{|F(x+i)|^{2}}{x^{2}+1 / 4} d x=\int_{-\infty}^{\infty} e^{-v}\left|\int_{0}^{e^{-v}} \varphi(u) d u\right|^{2} d v=\int_{0}^{1}\left|\int_{0}^{t} \varphi(u) d u\right|^{2} d t$.

It follows from (2) and the general Paley-Wiener theory that

$$
G(z)=F(z) \prod_{m \leqq n-1}\left(\frac{z+i(m+1 / 2}{z-i(m+1 / 2}\right)^{n}
$$

is in $P W$. Further, since $|x+i(m+1 / 2)|=|x-i(m+1 / 2)|$, (3) gives

$$
\frac{1}{2 \pi} \int_{-\infty}^{\infty}|G(x)|^{2} d x=1,
$$

and (4) becomes

$$
\int_{0}^{1}\left|\int_{0}^{t} \varphi(u) d u\right|^{2} d t=\frac{1}{2 \pi} \int_{-\infty}^{\infty} \frac{|G(x+i)|^{2}}{x^{2}+1 / 4} \prod_{m \leqq n-1}\left(\frac{x^{2}+(m-1 / 2)^{2}}{x^{2}+(m+3 / 2)^{2}}\right)^{n} d x .
$$

From (5), (6) and Lemma 6 we get

$$
\rho_{n}^{2}=\sup _{G \in P W} \frac{\int_{-\infty}^{\infty} \frac{|G(x+i)|^{2}}{x^{2}+1 / 4} \prod_{m \leqq n-1}\left(\frac{x^{2}+(m-1 / 2)^{2}}{x^{2}+(m+3 / 2)^{2}}\right)^{n} d x}{\int_{-\infty}^{\infty}|G(x)|^{2} d x} .
$$

But $\|G(x+i)\|_{L^{2}}^{2^{2}} \leqq G(x) \|_{L^{2}}^{2^{2}}$. Hence

$$
\begin{aligned}
\rho_{n}^{2} & \leqq \max _{x} \frac{1}{x^{2}+1 / 4} \prod_{m \leqq n-1}\left(\frac{x^{2}+(m-1 / 2)^{2}}{x^{2}+(m+3 / 2)^{2}}\right)^{n} \\
& =\max _{x} \frac{\left(x^{2}+1 / 4\right)^{2 n-1}}{\left(x^{2}+(n-1 / 2)^{2}\right)^{n}\left(x^{2}+(n+3 / 2)^{2}\right)^{n}}
\end{aligned}
$$


We note that the function $x=x(n)$ at which the max is taken on must go to infinity with $n$, for, if not, the upper bound thus obtained for $\rho_{n}$ would grossly violate the general lower bound theorem. This being the case,

$$
\rho_{n}<c \max _{x} \frac{x^{2 n-1}}{\left(x^{2}+n^{2}\right)^{n}}
$$

The max occurs at $x^{2}=2 n^{3}-n^{2}$, and the maximum value is $c\left(2 n^{3}-n^{2}\right)^{-1 / 2}(1-1 / 2 n)^{n}$, which tends to $c /(2 e)^{1 / 2} n^{3 / 2}$, giving the desired upper bound.

Choosing $G(z)=1 /\left(z+i n^{3 / 2}\right)$ we get from (7) that

$$
\begin{aligned}
\rho_{n}^{2} & \geqq \frac{\int_{-\infty}^{\infty} \frac{c}{x^{2}}\left(\frac{x^{2}}{x^{2}+n^{2}}\right)^{2 n} \frac{d x}{x^{2}+n^{3}}}{\int_{-\infty}^{\infty} \frac{d x}{x^{2}+n^{3}}} \\
& \geqq \frac{n^{3 / 2}}{\pi} \int_{n^{3 / 2}}^{2 n^{3 / 2}} \frac{c^{\prime}}{x^{2}}\left(\frac{x^{2}}{x^{2}+n^{2}}\right)^{2 n} \frac{d x}{x^{2}+n^{3}} \\
& \geqq \frac{c^{\prime}}{\pi 2 e^{2} n^{3}} \int_{1}^{2} \frac{d u}{u^{2}+1}=\frac{c^{\prime \prime}}{n^{3}} .
\end{aligned}
$$

Our interest will now, temporarily, be turned from linear combinations of $x^{k}$, $k=0,1, \ldots, n$ as approximating functions to approximation with linear combinations of $x^{k / q}, k=0,1,2, \ldots, n q$, on the interval $Q_{1}$. We refer to the latter space and the corresponding degree of approximation as $P_{n q}^{*}$ and $\rho_{n q}^{*}$.

Since $P_{n} \subset P_{n q}^{*}$, it follows from Jackson's theorem that there is a $c_{1}$ such that $\rho_{n q}^{*} \leqq c_{1} / n q$. By the general lower bound theorem, there is a $c_{2}$ such that $\rho_{n q}^{*} \geqq c_{2} / n$. The following result, in addition to being of independent interest, will be used in the proof of Theorem 4 .

THEOREM 3. There exist constants $c_{1}, c_{2}>0$ such that $c_{1} / n q^{1 / 2} \leqq \rho_{n q}^{*} \leqq c_{2} / n q^{1 / 2}$.

Proof. By Lemma 7,

$$
\rho_{n q}^{* 2}=\sup _{F \in P W} \frac{\int_{-\infty}^{\infty} \frac{|F(x+i)|^{2}}{x^{2}+1 / 4} \prod_{k=0}^{n q} \frac{x^{2}+(k / q-1 / 2)^{2}}{x^{2}+(k / q+3 / 2)^{2}} d x}{\int_{-\infty}^{\infty}|F(x)|^{2} d x},
$$

while, by Lemma 8 ,

$$
\rho_{n q}^{* 2} \leqq M(\Lambda), \text { where } \Lambda=\{k / q \mid k=0,1, \ldots, n q\} .
$$

But, by cancellation,

(10) $M(\Lambda)=\max _{x}\left(x^{2}+1 / 4\right)^{-1} \prod_{k=0}^{2 q-1}\left(x^{2}+(k / q-1 / 2)^{2}\right) \prod_{j=q n-2 q+1}\left(x^{2}+(j / q+3 / 2)^{2}\right)^{-1}$.

By (9) and by the general lower bound theorem, $M$ cannot fall off faster than $c / n^{4}$. The point $x=x(n, q)$ where the max is taken on must go to infinity with $n$ or $q$, 
for, if not, (10) would yield $M=O\left(1 / n^{2 q n}\right)$. We now have, from (10), that there exist positive constants $k_{1}, k_{2}$ such that

$$
k_{1} M \leqq \frac{1}{x^{2}}\left(\frac{x^{2}}{x^{2}+n^{2}}\right)^{2 q} \leqq k_{2} M .
$$

The max in (11) is determined to be at $x^{2}=2 q n^{2}-n^{2}$, yielding $M=O\left(1 / n^{2} q\right)$. Thus, from (9) we have

$$
\rho_{n q}^{*}=O\left(1 / n q^{1 / 2}\right) .
$$

Choosing $F(z)=1 /\left(z+i n q^{1 / 2}\right)$, we have, from (8) and (11),

$$
\begin{aligned}
\rho_{n q}^{*^{2}} & \geqq \frac{\int_{-\infty}^{\infty} \frac{c}{x^{2}}\left(\frac{x^{2}}{x^{2}+n^{2}}\right)^{2 q} \frac{d x}{x^{2}+n^{2} q}}{\int_{-\infty}^{\infty} \frac{d x}{x^{2}+n^{2} q}} \\
& \geqq \frac{n q^{1 / 2}}{\pi} \int_{n q^{1 / 2}}^{2 n q^{1 / 2}} \frac{c}{x^{2}}\left(\frac{x^{2}}{x^{2}+n^{2}}\right)^{2 q} \frac{d x}{x^{2}+n^{2} q} \\
& \geqq \frac{c}{\pi n q^{1 / 2}}(1+1 / q)^{-2 q} \int \frac{d x}{x^{2}+n^{2} q} \geqq \frac{c^{\prime}}{n^{2} q},
\end{aligned}
$$

which, together with (12), proves the theorem.

Let $Q$ be the curve $y=x^{p / q}, p, q$ relatively prime, $\delta \leqq x \leqq 1$. Consider the family of all such $Q$. Since these curves are algebraic, Theorem 1 implies $c_{1} / n \leqq \rho_{n} \leqq c_{2} / n$. However, for each $Q$ in the family, the constants, $c_{1}, c_{2}$, although independent of $n$, might well depend on which particular curve from the family is under consideration, i.e, $c_{1}$ and/or $c_{2}$ may be nontrivial functions of $p$ and/or $q$. We shall now concern ourselves with more precise estimates for $\rho_{n}$-estimates that will yield the order of magnitude as a function of which $Q$ from the family is under consideration. The following result in this direction will be crucial to the proof of Theorem 5.

THEOREM 4. Let $Q$ be the set $\left(x, x^{p / q}\right), \delta \leqq x \leqq 1, p, q$ relatively prime. Then there exist $c_{1}, c_{2}>0$ such that, in the $L^{2}$ norm, $c_{1} / n q^{1 / 2} \leqq \rho_{n} \leqq c_{2} / n q^{1 / 2}$ for $n, p, q$ such that $n \geqq q$.

Proof. We note that for $y=x^{p / q}$, approximating with $n$th degree polynomials in $x$ and $y$ is the same as approximating with $x^{i+j p / q}, 0 \leqq i, j \leqq n$.

Let $f^{*}(x)=f\left(x, x^{p / q}\right)$ be defined for $\delta \leqq x \leqq 1$. Now

$$
\left\|f\left(x, x^{p / q}\right)-p_{n}\left(x, x^{p / q}\right)\right\|=\left\|f^{*}(x)-\sum a_{i j} x^{i+j p / q}\right\| .
$$

Also, the slope of $f^{*}(x)$,

$$
\frac{\left\|f^{*}(y)-f^{*}(x)\right\|}{d(x, y)}=\frac{\left\|f\left(y, y^{p / q}\right)-f\left(x, x^{p / q}\right)\right\|}{d\left(\left(x, x^{p / q}\right),\left(y, y^{p / q}\right)\right)} \cdot \frac{d\left(\left(x, x^{p / q}\right),\left(y, y^{p / q}\right)\right)}{d(x, y)} \leqq\left(1+m^{2}\right)^{1 / 2},
$$

where $m$ is the maximum slope for $y=x^{p / q}$ on $[\delta, 1]$. Since $m<1 / \delta$, we have 
$\left\|f^{*}(y)-f^{*}(x)\right\| \leqq M|y-x|, M=\left(1+1 / \delta^{2}\right)^{1 / 2}$. Thus $f^{*}(x) / M$ is in $K(\delta, 1)$. Hence the assertion of our theorem is equivalent to the existence of $c_{1}, c_{2}$ such that

$$
\frac{c_{1}}{n q^{1 / 2}} \leqq \max _{f \in K(\delta, 1)} \min _{\left\{a_{i j}\right\}}\left\|f(x)-\sum_{i, j=0}^{n} a_{i j} x^{i+j p / q}\right\| \leqq \frac{c_{2}}{n q^{1 / 2}}
$$

First, the left inequality in (13) will be established. Define

$$
\rho_{n q}^{*}(a, b) \max _{f \in K(a, b)} \min _{\left\{a_{k}\right\}}\left\|f(x)-\sum_{k=0}^{n q} a_{k} x^{k / q}\right\| .
$$

LEMMA 12. $\rho_{2 n q}^{*}(\delta, 1) \geqq(1-\delta) \rho_{2 n q}^{*}(0,1)$.

Proof. With respect to $\rho_{2 n q}^{*}(0,1)$, consider the coefficients $\left\{b_{k}\right\}$ for the function, $g$, that maximizes. Then

$$
\left\|g(x)-\sum_{0}^{2 n q} b_{k} x^{k / q}\right\|=\rho_{2 n q}^{*}(0,1) .
$$

Let $f(x)=(1-\delta) g((x-\delta) /(1-\delta)) . f(x)$ is in $K(\delta, 1)$. Now

$$
\sum_{0}^{2 n q} a_{k} x^{k / q}=\sum(1-\delta) b_{k}((x-\delta) /(1-\delta))^{k / q}
$$

is the best approximating polynomial to $f(x)$ on $[\delta, 1]$. For, suppose the contrary, i.e.,

$$
\left\|\sum c_{k} x^{k / q}-f(x)\right\|<\left\|\sum(1-\delta) b_{k}((x-\delta) /(1-\delta))^{k / q}-f(x)\right\|
$$

on $[\delta, 1]$. Then, on $[0,1]$,

$$
\begin{aligned}
\left\|\sum c_{k}((1-\delta) x+\delta)^{k / q}-f((1-\delta) x+\delta)\right\| & \leqq\left\|\sum(1-\delta) b_{k} x^{k / q}-f((1-\delta) x+\delta)\right\| \\
& \leqq\left\|\sum(1-\delta) b_{k} x^{k / q}-g(x)\right\|,
\end{aligned}
$$

contrary to the assumption that the $b_{k}$ minimized for $g$. Hence,

$$
\begin{aligned}
\rho_{2 n q}^{*}(\delta, 1) & \geqq\left\|\sum(1-\delta) b_{k}((x-\delta) /(1-\delta))^{k / q}-f(x)\right\| \quad \text { on }[\delta, 1] \\
& =(1-\delta)\left\|\sum b_{k}((x-\delta) /(1-\delta))^{k / q}-g((x-\delta) /(1-\delta))\right\| \quad \text { on }[\delta, 1] \\
& =(1-\delta)\left\|\sum b_{k} x^{k / q}-g(x)\right\| \quad \text { on }[0,1] \\
& =(1-\delta) \rho_{2 n q}^{*}(0,1),
\end{aligned}
$$

and Lemma 12 is established. Now,

$$
\max _{f \in K(\delta, 1)} \min _{\left\{a_{i j\}}\right\}}\left\|f(x)-\sum a_{i j} x^{i+j p / q}\right\| \geqq \rho_{2 n q}^{*}(\delta, 1) \geqq(1-\delta) \rho_{2 n q}^{*}(0,1) \geqq \frac{c}{n q^{1 / 2}},
$$

the last inequality following from Theorem 3.

We now prove the upper bound in (13). Let $f$ be extended, as a contraction, to $[0,1]$. Lemma 8 now reduces the proof of the desired inequality to showing that

$$
M(\Lambda)<c / n^{2} q, \text { where } \Lambda=\{i+j p / q, 0 \leqq i, j \leqq n\} .
$$


Let $x=x(n, q)$ be the point $x$ at which the max in $M(\Lambda)$ is taken on. As in the proof of Theorem 2, $x(n, q)$, for fixed $q$, must tend to infinity with $n$. Observe that $\left(x^{2}+(\lambda-1 / 2)^{2}\right) /\left(x^{2}+(\lambda+3 / 2)^{2}\right)$ is an increasing function of $\lambda$ for $\lambda>4\left(1+x^{2}\right)^{1 / 2}-2$, and decreasing for $\lambda$ less than this quantity. Let $q$ be fixed. We consider two possible cases:

Case I. $1 / x(n, q)=O(1 / n)$.

Then there is some $c$ such that $\left(x^{2}+(\lambda-1 / 2)^{2}\right) /\left(x^{2}+(\lambda+3 / 2)^{2}\right)$ is decreasing for all $\lambda<c n$. Let $\Lambda_{0}$ be $\{\lambda \in \Lambda \mid \lambda<c n\} . M\left(\Lambda_{0}\right) \geqq M(\Lambda)$. The facts that

$$
\left(x^{2}+(\lambda-1 / 2)^{2}\right) /\left(x^{2}+(\lambda+3 / 2)^{2}\right)<1,
$$

that this quotient is a decreasing function of $\lambda$, and that, by Lemma 11 , there are at least $\min (k, q-1)$ members of $\Lambda_{0}$ between $k$ and $k+1$ for $k+1<c n$ imply that $M\left(\Lambda_{1}\right)>M\left(\Lambda_{0}\right)$, where

$$
\Lambda_{1}=\{i+j / q, 0 \leqq i \leqq[c n], 0 \leqq j \leqq \min (i, q)\} .
$$

That is,

$$
\begin{aligned}
& \Lambda_{1}=\{0 \text {, } \\
& 1, \quad 1+1 / q \\
& 2, \quad 2+1 / q, \quad 2+2 / q, \\
& 3, \quad 3+1 / q, \quad 3+2 / q, \quad 3+3 / q, \\
& 4, \quad 4+1 / q, \quad 4+2 / q, \quad 4+3 / q \text {, } \\
& \vdots \quad \vdots \quad \vdots \quad \vdots \\
& q-1, \quad q-1+1 / q, \quad q-1+2 / q, \quad \ldots, \quad q-1+(q-1) / q, \\
& q, \quad q+1 / q, \quad q+2 / q, \quad \ldots, \quad q+(q-1) / q \text {, } \\
& [c n-1],[c n-1]+1 / q, \quad[c n-1]+2 / q, \quad \ldots, \quad[c n-1]+(q-1) / q\} .
\end{aligned}
$$

By the cancellation of the terms in the product (all but the first two and last two entries in each column cancel) we have

$$
M\left(\Lambda_{1}\right)=\max _{x} \frac{1}{x^{2}+\frac{1}{4}} \prod_{k=0}^{q} \frac{x^{2}+(k+k / q-1 / 2)^{2}}{x^{2}+([c n]+k / q+1 / 2)^{2}} \cdot \frac{x^{2}+(k+k / q+1 / 2)^{2}}{x^{2}+([c n]+k / q+3 / 2)^{2}}
$$

which is of the order of

or

$$
\max _{x} \frac{1}{x^{2}}\left(\prod\left(1-\frac{c^{2} n^{2}-k^{2}}{x^{2}+c^{2} n^{2}}\right)\right)^{2}<\max _{x} \frac{1}{x^{2}} \exp \left(-2 \sum^{q} \frac{c^{2} n^{2}-k^{2}}{x^{2}+c^{2} n^{2}}\right)
$$

$$
\max _{x} \frac{1}{x^{2}} \exp \left(-\frac{2 c^{2} n^{2} q-q^{3} / 3}{x^{2}+n^{2}}\right)
$$

But the maximum (away from the origin) is taken at $x^{2}=n^{2}\left(2 c q^{2}-1\right)-q^{3} / 3$, where we get $M\left(\Lambda_{1}\right)=O\left(1 / q n^{2}\right)$, and the estimate is established for Case I. 
Case II. We now suppose that $1 / x(n, q)$ is not $=O(1 / n)$. Then there is a subsequence of $\{n\}$ for which $x(n, q)=O(n)$. Let $\Lambda_{2}=\{\lambda \in \Lambda \mid \lambda>[4 x]\} . M\left(\Lambda_{2}\right)>M(\Lambda)$. Now, that $\left(x^{2}+(\lambda-1 / 2)^{2}\right) /\left(x^{2}+(\lambda+3 / 2)^{2}\right)$ is an increasing function of $\lambda$ bounded by 1 implies, in view of Lemma 11 , that $M\left(\Lambda_{3}\right)>M\left(\Lambda_{2}\right)$, where

$$
\Lambda_{3}=\{i-j / q \mid[4 x] \leqq i \leqq n, 0 \leqq j \leqq \min (i, q)\} .
$$

That is,

$$
\begin{array}{ccccc}
\Lambda_{3}=\{[4 x]-\min ([4 x], q-1) / q, & \ldots, & {[4 x]-2 / q,} & {[4 x]-1 / q,} & {[4 x],} \\
{[4 x]+1-\min ([4 x]+1, q-1) / q,} & \ldots, & {[4 x]+1-2 / q,} & {[4 x]+1-1 / q,} & {[4 x]+1,} \\
{[4 x]+2-\min ([4 x]+2, q-1) / q,} & \ldots, & {[4 x]+2-2 / q,} & {[4 x]+2-1 / q,} & {[4 x]+2,} \\
\vdots & & \vdots & \vdots & \vdots \\
n-(q-1) / q, & \ldots, & n-2 / q, & n-1 / q, & n\} .
\end{array}
$$

By cancellation of terms in the product, we have

$$
M\left(\Lambda_{3}\right) \leqq \max _{x} \frac{1}{x^{2}+\frac{1}{4}} \prod_{k=0}^{\min ([4 x, q])} \frac{x^{2}+([4 x]-k / q-1 / 2)^{2}}{x^{2}+(n-k / q+1 / 2)^{2}} \cdot \frac{x^{2}+([4 x]-k / q-3 / 2)^{2}}{x^{2}+(n-k / q-1 / 2)^{2}} .
$$

Thus, for the subsequence of $n$ for which $x=O(n)$, we have

$$
M\left(\Lambda_{3}\right)=O\left(c x^{2} / n^{2}\right)^{\alpha}, \quad \alpha=\min ([4 x], q) .
$$

Hence, for these values of $n, M(\Lambda)<M\left(\Lambda_{3}\right)<1 / n^{5}$, which is impossible by the lower bound theorem. Thus Case II cannot occur and the theorem is established.

We are now in a position where we can give a proof of Theorem 5: Consider all $n$ for which there exist $p$ such that $|\alpha-p / n|<1 / n^{2}(3 /(1-\delta))^{2 n^{2}}$. This is a subsequence $n_{i}$ of $n$, and defines our subsequence $\rho_{n_{i}}$ of $\rho_{n}$. For convenience of notation, the subscripts will be dropped, i.e., $n_{i}$ will be referred to as $n$. It will be shown that

$$
\varepsilon c_{1} / 2 n^{3 / 2} \leqq \rho_{n} \leqq 2 c_{2} / \varepsilon n^{3 / 2} .
$$

(Here $c_{1}$ and $c_{2}$ are the same constants as those in Theorem 4.)

Extend $f(x, y)$, now defined as a contraction on $y=x^{\alpha}$, to be a contraction on the square $0 \leqq x, y \leqq 1$. That this can be done follows, again, from the corollary to Lemma 3. Consider the curve $y=x^{p / n}$. Then

$$
\left|x^{\alpha}-x^{p / n}\right|<1 / \varepsilon n^{4}(3 /(1-\delta))^{2 n^{2}} .
$$

By Theorem 4 , there exists $p_{n}(x, y)$ such that

$$
\left\|p_{n}\left(x, x^{p / n}\right)-f\left(x, x^{p / n}\right)\right\| \leqq c_{2} / n^{3 / 2} .
$$

Now,

$$
\begin{aligned}
\left\|p_{n}\left(x, x^{\alpha}\right)-f\left(x, x^{\alpha}\right)\right\| \leqq & \left\|p_{n}\left(x, x^{\alpha}\right)-p_{n}\left(x, x^{p / n}\right)\right\| \\
& +\left\|p_{n}\left(x, x^{p / n}\right)-f\left(x, x^{p / n}\right)\right\| \\
& +\left\|f\left(x, x^{p / n}\right)-f\left(x, x^{\alpha}\right)\right\|
\end{aligned}
$$


Since $f$ is a contraction, it follows from (15) that

$$
\left\|f\left(x, x^{p / n}\right)-f\left(x, x^{\alpha}\right)\right\| \leqq 1 / \varepsilon n^{4}(3 /(1-\delta))^{2 n^{2}} .
$$

Now,

$$
\begin{aligned}
\left\|p_{n}\left(x, x^{\alpha}\right)-p_{n}\left(x, x^{p / n}\right)\right\| & \leqq \max _{\delta \leqq x \leqq 1}\left|p_{n}\left(x, x^{\alpha}\right)-p_{n}\left(x, x^{p / n}\right)\right| \\
& \leqq \max _{\delta \leqq x \leqq 1} \sum^{n}\left|\left(\frac{d^{k}}{d y^{k}} p_{n}(x, y)\right)_{y=x^{p / n}}\right|\left|x^{\alpha}-x^{p / n}\right|^{k},
\end{aligned}
$$

by Taylor's Theorem. But this is

$$
<\frac{1}{\varepsilon n^{4}(3 /(1-\delta))^{2 n^{2}}} \sum\left|\frac{d^{k}}{d y^{k}} p_{n}(x, y)\right|_{y=x^{p / n}}
$$

by (15). But, by Lemma 10 , the coefficients of $p_{n}\left(x, x^{p / n}\right)$ are bounded by $(3 /(1-\delta))^{2 n^{2}}$. Hence, we have

$$
\left\|p_{n}\left(x, x^{\alpha}\right)-p_{n}\left(x, x^{p / n}\right)\right\|<1 / \varepsilon n^{2} .
$$

Substitution of (16), (18), and (19) in (17) yields the upper bound asserted in (14). To establish the lower bound, we note that

$$
\begin{aligned}
&\left\|p_{n}\left(x, x^{\alpha}\right)-f\left(x, x^{\alpha}\right)\right\|+\left\|p_{n}\left(x, x^{\alpha}\right)-p_{n}\left(x, x^{p / n}\right)\right\|+\left\|f\left(x, x^{p / n}\right)-f\left(x, x^{\alpha}\right)\right\| \\
& \geqq\left\|p_{n}\left(x, x^{p / n}\right)-f\left(x, x^{p / n}\right)\right\|,
\end{aligned}
$$

and hence that by (18), (19) and Theorem 4,

$$
\left\|p_{n}\left(x, x^{\alpha}\right)-f\left(x, x^{\alpha}\right)\right\| \geqq \frac{c_{1}}{n^{3 / 2}}-\frac{1}{n^{4}}-\frac{1}{n^{2}} \geqq \frac{c_{1}}{2 n^{3 / 2}} . \quad \text { Q.E.D. }
$$

\section{BIBLIOGRAPHY}

1. S. Bernstein, Leçons sur les propriétés extrémales et la meilleure approximation des fonctions analytiques d'une variable réelle, Gauthier-Villars, Paris, 1926.

2. Yu. A. Brudnji, "Constructive characteristics of functions given on certain perfect sets on the real axis," pp. 122-126, in Investigation on contemporary problems of the constructive theory of functions, edited by V. I. Smirnov, Fizmatgiz, Moscow, 1961.

3. D. Jackson, The theory of approximation, Amer. Math. Soc. Colloq. Publ., Vol. 11, Amer. Math. Soc., Providence, R. I., 1930.

4. G. G. Lorentz, Approximation of smooth functions, Bull. Amer. Math. Soc. 66 (1960), 124-125.

5. D. J. Newman, A Müntz-Jackson theorem, Amer. J. Math. 87 (1965), 940-944.

6. D. J. Newman and H. S. Shapiro, "Jackson's theorem in higher dimensions," pp. 208219, in On approximation theory, proceedings of the conference at Oberwolfach, 1963, Birkhäuser, Basel, 1964.

7. R. E. A. C. Paley and N. Wiener, Fourier transforms in the complex domain, pp. 1-13, Amer. Math. Soc. Colloq. Publ., Vol. 19, Amer. Math. Soc., Providence, R. I., 1934; reprint 1960.

\footnotetext{
YeSHIVA UNIVERSITY, New York, New York

TEMPLE UNIVERSITY,

Philadelphia, Pennsylvania
} 